\title{
On the limits of cultural relativism as a debiasing method
}

\section{David Teira | UNED}

I analyse cultural relativism as a methodological strategy to correct for ethnocentric biases in anthropological fieldwork. I discuss the format debiasing norms may adopt (rules or standards) depending on whether a discipline has a causal or interpretative outlook. Boas and his school advocated for an interpretative approach to ethnographic fieldwork, in which cultural relativism was implemented as a standard ("Only culturally unbiased reports are admissible") to be interpreted by expert third parties. Legitimate as it may be as a debiasing method, it does not allow anthropologists to adjudicate their debates on biases in their ethnographic record. 


\section{Cultural Relativism as a Debiasing Procedure}

Philosophers of science have extensively debated cultural relativism for already more than half a century (Koskinen 2019). The consensual view is that it is only legitimate as a method to control for ethnocentrism in anthropological fieldwork, and objectionable in every other respect (e.g., about human rights). For gathering data in the field, anthropologists usually rely on participant observation, taking active part in the ways of life under study while keeping a record of what goes on. The methodological challenge of participant observation is that there is no pre-established study protocol with closed research questions and data-collection procedures: the questions addressed and the necessary data are usually articulated throughout the observation process (Zahle 2013).

In this context, cultural relativism is a prescription for the anthropologist to suspend her own judgments in order to prevent the contamination of her fieldwork records with her native culture's values. From a methodological standpoint, ethnocentrism would be a potential bias for the anthropologist and cultural relativism the debiasing device that should control for it. I am going to argue that cultural relativism is a legitimate but ineffective bias correction, as endless controversies among anthropologists illustrate. More precisely, cultural relativism is a methodological prescription articulated in a way that makes impossible to adjudicate whether the data are actually biased and in which way they should be corrected.

I will discuss first, in the following section, how the cognitive bias of the research participants interferes in the data gathering process, and how researchers in different scientific fields have learnt to control for these biases with several rules of thumb. Drawing on an 
established distinction among legal philosophers, I will argue next that these rules of thumb can be rendered into methodological prescriptions with two different formats: rules and standards. If biases are understood as causal interferences in the data gathering process, debiasing norms are better articulated as rules -norms of the conditional form if $X$, then $Y$. Interpretative methods, like participant observation in anthropology, do not fit well with a causal understanding of biases, and debiasing norms are instead articulated as standards general prescriptions that experts should interpret on a case by case basis.

In section 3, I will discuss how cultural relativism emerged within a non-causal approach to anthropological fieldwork inaugurated by Franz Boas and his school. They established it as a professional norm among anthropologists with a view to fighting ethnocentrism, articulating it as a standard of the form "Only culturally unbiased reports are admissible". Despite the institutional success of this norm (widely endorsed by the major scholarly societies in the field), anthropologists have not agreed so far on how to interpret it in order to adjudicate conflicts about biased evidence. In section 4, I will discuss several debates on ethnocentrism in a leading anthropology journal to show cultural relativism is a completely ineffective methodological norm when it comes to close a controversy. In the fifth and final section, I will discuss why, despite its lack of effectiveness, cultural relativism may not hinder the progress of anthropology. On the one hand, it may work better as a guideline for correct reporting than an actual debiasing method. On the other hand, since most anthropologists do not aggregate evidence to ground far-reaching theories, they may not need to standardize their controls for bias (as it happens in other scientific fields). 


\section{Biases: Causes or Misinterpretations}

Let me informally introduce the concept of cognitive bias. When we speak of an unbiased estimator in statistics, we refer to the difference between this estimator's expected value and the true value of the parameter being estimated. If we are interested in the latter, we want this difference to be zero. The problem with most sources of evidence is that we do not know the true value of the variable under investigation: the evidence collected should yield our best approximation. However, if there is some systematic interference in the data collection process, we will say that the evidence is biased and will not ground sound inferences about the truths under investigation. How can we know, without begging the principle, that there is a bias without knowing the true value?

Psychologists have documented a general tendency to incur all sorts of unintentional biases in our dealings with empirical data. Knowing the true value a given cognitive process should yield, psychologists have constructed many experimental setups in which subjects, unaware of the true value, try to reach it drawing on the available evidence. One particularly interesting instance is our general selectivity in the treatment of evidence: we tend to handle data in a way that, for instance, does not give equal weight to evidence favourable and unfavourable for our preferences. This is called the confirmation bias and it is a solid experimental regularity. Different psychological mechanisms have been hypothesized in order to account for confirmation biases (Weber and Johnson 2009): e.g., affective influences on cognitive operations on beliefs; limitations of our information-processing capacities; conflicts between conscious and unconscious information-processing mechanisms, etc. However, like with most other biases, there is no agreement on the on the explanatory mechanism for the 
confirmation bias. And, consequently, there is nothing like a consensual unified theory about cognitive biases. What psychologists have is a catalogue of experiments on biases probing different cognitive resources (e.g., attention, encoding and evaluation of data, memory). In these experiments, biases emerge regularly as systematic deviations from a benchmark value. From the standpoint of psychology, biases are thus solid experimental regularities.

Scientists suffer from cognitive biases when they gather evidence, developing methodological checks to correct them (Fugelsang et al. 2004). Experimentalists in different fields have detected practices that yield systematic deviations from what turns out to be the true value. By trial and error, they have also found methods for the correction of such biases in their data gathering processes. Think for instance of blinding: masking treatments in an experiment so that the participants cannot ascertain which one they are giving or receiving. Inquisitors apparently used blinding in witchcraft trials, but it was Lavoisier who systematized it as an experimental control at the end of the $18^{\text {th }}$ century. Lavoisier was part of a committee testing the therapeutic efficacy of animal magnetism, a controversial intervention advocated by the German physician Franz Anton Mesmer. Lavoisier suggested to blind patients in order to separate the reactions caused by magnetism itself from those elicited by the therapist through other means. Lavoisier, like the inquisitors before him, did not have a theory to account for the cognitive mechanisms that blinding defused. They just tried it and saw that blinding showed that patients did not react to animal magnetism if they did not know they were receiving it.

In a separate paper (Teira 2016) I have discussed under which conditions these rules of thumb become justified methodological norms: debiasing methods warrant the neutrality of 
the experiment regarding an agreed list of potential interferences (such as the preferences of the participants). My claim is now that depending on how cognitive biases are understood in a discipline, the debiasing rules of thumb will yield methodological norms of at least two kinds: rules and standards. This is a legal distinction that goes as follows (Luppi and Parisi 2011). Rules are norms that can be rendered into conditionals of the form if $\mathrm{X}$, then $\mathrm{Y}$ : "Dogs are not allowed in the bar" can be paraphrased as "If X is a dog, then X is not allowed in the bar". Standards are instead norms open to interpretation: "Only good dogs are allowed in the bar" requires that somebody assess the character of the dog, before letting it in.

I contend that if cognitive biases are understood as causal interferences in the data gathering process, the debiasing norms will be better articulated as rules. In experimental fields such as physics or medicine, biases are usually understood as uncontrolled causal factors with an influence in the intervention under study (Staley 2004; Chalmers 2006). The understanding of causality may be rough. In medicine, the causes of disease or the statistical analysis of causation about 150 years old now. Yet, for centuries, medical researchers have shared a rough grasp of the differences made by the various elements in a treatment and, in particular, by the preferences of the physicians and patients. These preferences introduce cognitive biases when testing treatments: as we already saw, blinding emerged to control for the patients' preferences and make sure that they had no role in the ascertainment of the treatment effect. Randomization was introduced in the $18^{\text {th }}$ century as a simple correction for the selection bias introduced by physicians who allocated treatments depending on the characteristics of the patients. Debiasing methods warrant a fair test, facilitating a like with 
like comparison between treatments in which the preferences of any participant play no role in the data generation process.

If cognitive biases are understood as causal confounders, debiasing methods will better adopt the format of a conditional rule: if, at any given stage in the experiment, there is a potential risk of bias, the relevant correction should be implemented. E.g., if there is a risk of a physician allocating treatments according to her preferences, randomize; if there is a risk of a patient reacting to a treatment according to his preferences, blind the treatment. Why a rule better than a standard? The economic analysis of law has shown that, under certain assumptions, rules are more effective than standards in order to prevent conflicts of interest (Fon and Parisi 2007). Standards require interpretation and are therefore open to self-serving implementations; rules are comparatively automatic: if the antecedent condition occurs, a definite action should be triggered.

However, if biases in the data gathering process are not understood in causal terms, standards may provide an easier rendition. This is the case of the interpretative social sciences, where instead of searching for causal regularities, research aims at capturing singular phenomena in mostly qualitative terms. The biases interfering in the data gathering process are here understood as distortions in the interpretative process: e.g., researchers misinterpreting the data due to their own prejudices. As we are going to discuss next, ethnocentrism in anthropology is perhaps the most prominent example of this understanding of bias. For this sort of cognitive bias, there is no obvious hard trigger, since research is not understood causally: the distortion is usually perceived by a competent third party. Therefore, 
the debiasing methods are better rendered as standards to be interpreted by an expert, as we are going to illustrate next with cultural relativism.

\section{Cultural Relativism as a Debiasing Standard}

Historically, there have been many different rules of thumb warning about the different cultural perspectives in a report -e.g., Xenophanes (570-480BC) warned about different people attribute their own characteristics to their gods. However, the transformation of these rules of thumb into a methodological norm came only with the institutional rise of anthropology as a scientific field, a process that took place mainly in the US during the first half of the $20^{\text {th }}$ century. Franz Boas, the leading advocate for cultural relativism, was also one of the founding fathers of the discipline, contributing through his students to establish many graduate programs in the US. In these programs, cultural relativism became one of the defining traits of the profession.

Franz Boas systematized this rule of thumb as a professional standard throughout his life-long debate against a hierarchical view of cultures, typical of so many $19^{\text {th }}$ century anthropologists (Hatch 1983). Drawing on extensive ethnographic evidence, Boas used human variability to argue against any causal inferences in anthropology and, in particular, against those who ranked biological or cultural traits in terms their purported superiority. Underlying the hierarchy there would be a causal genealogy, in which the simpler forms would precede and bring about the more complex. For Boas, instead, there were neither laws in culture nor unambiguous categories capturing different instantiations of a given phenomenon in different cultural setups. There would not be either statistical tool powerful 
enough to decompose human variability into specific causal factors with separate contributions to either biological or cultural phenomena (Boas [1936] 1940, 309). According to Boas, whatever general structures there could be in a given culture, the individuals operating from within constantly transform them. Even external interventions alter the phenomena under study. For Boas, this is why the ideal of experimental control could not be achieved when dealing with cultural phenomena (Boas [1932] 1940, 256) The anthropologist should rely instead on participant observation: spend time in a culture and create an extensive record of its different practices, trying to capture what's specific to them.

In this context, Boas warned against those anthropological analyses that imposed their own cultural categories on their topic. He wanted “freedom from cultural prejudice”. Boas' disciples dubbed this prejudice ethnocentrism, "the point of view that one's way of life is to be preferred to all others" (Herskotvits [1955] 1973). Boas gave no methodological recipe for the correction of this prejudice, other than "the intensive study of foreign cultures of fundamentally distinctive types" (Boas and Benedict 1938, 685). For Boas and his school, extended fieldwork, thorough learning of the local language, detailed notes written up daily while in the field, and the prohibition on premature or uncritical generalizations seemed to be the natural remedy for ethnocentrism.

The Boasian school systematized this general warning against prejudice into cultural relativism. Perhaps the most popular formulation is Melville Herskovits': “Judgments are based on experience, and experience is interpreted by each individual in terms of his own enculturation." (Herskotvits [1955] 1973, 15). Like Boas, Herskovits never discussed the psychological mechanisms triggering ethnocentrism, but he also praised culturally immersive 
fieldwork seemed as the best correction for ethnocentrism (Herskotvits [1960] 1973, 246). It was necessary to engage with the subjects under study until the anthropologist could grasp individuality beyond any stereotype.

Unlike in those experimental fields in which data are gathered through controlled interventions, the Boasians relied instead on participant observation. For them, biases could not be causal confounders, but rather interpretative prejudices. Rather than targeting any particular cognitive mechanism triggering them, they opted for warnings in the form of a standard like: "Only culturally unbiased reports are admissible" -or, using Herskovits' own terms: "Accept only reports without judgments based on the experience of the anthropologist's own enculturation". Third party experts would ultimately decide on whether the resulting ethnography had any trace of the author's cultural prejudices. Ethnocentrism, after all, would be a cultural phenomenon for which there would be no single causal structure to correct and detecting it is equally a matter of interpretation.

The success of this norm among US anthropologist was due mostly to the preeminence of Boas and his school in articulating and staffing the first graduate programmes in the country. Historians of the social sciences have sometimes argued that this professional aspiration to a bias-free discipline was a rhetorical device: scientist vindicated their impartiality to better advise the policy-makers. Instead, thanks to their fight against ethnocentrism, Boasian anthropologists became a leading critical voice on US foreign policy, so it is difficult to find any professional self-serving interest in cultural relativism. What we find instead among US anthropologists is an incensed disciplinary debate on the existence of a hierarchy of racial and cultural traits. The presentation of ethnographic evidence contaminated 
with ethnocentric biases provided, for the Boasians, a biased confirmation of such hierarchies. In order to settle the debate, it was necessary to agree on what counted as unprejudiced evidence for the profession. Whereas debiasing rules successfully settled these debates on the neutrality data in experimental fields, I want to defend next that if cultural relativism ever contributed to the professional consensus of anthropologists, it was never because they agreed on how to use it as a debiasing standard.

\section{What Counts as Unbiased Anthropological Data?}

Debiasing rules (of the form "If X, then Y") anticipate all those point in the data gathering process in which a bias may interfere and prescribe a definite action to prevent it. Methodological standards of the form "Only culturally unbiased reports are admissible" are open to interpretation: what counts as a bias and who should decide whether it is affecting the report? The problem is, of course, that there is no single source of authority among anthropologists to decide whether the standard has been breached. In fact, anthropologists have been debating for decades how to balance the authority of the different informants within a group. Should they be the oldest non-Westernized members of a group? (Herskotvits [1958] 1973, 88) himself suggested instead to "observe a wide range of persons in as many situations as possible", independently of age, gender, etc. Methodologists of participant observation recommended relying not just on informants, but also on anthropological theory, introspective examination, etc. However, these recommendations rarely disambiguate the standard of cultural relativism precisely enough to establish whether it has actually been breached. In other words, formulated as a standard, cultural relativism does not allow anthropologists to reach a consensus on the quality of an actual dataset. 
In order to make my case, I have explored the 329 mentions of the keyword ethnocentrism featuring in the JSTOR collection of the American Anthropologist, the journal of the American Anthropological Association. I have focused on accusation of bias raised against particular papers and the exchanges that followed in the commentaries section. The number of these exchanges is low, as one may expect from a scholarly journal in which editors and reviewers should have screened off the grossest forms of ethnocentrism. Yet, the remaining controversies illustrate my point that invoking the universal agreement on the standard of cultural relativism does not help much in deciding whether it has been breached. I will provide just a couple of examples.

(Balzer 1981) presents a theory about the rituals of gender identity among the Siberian Khanty within a general discussion of religious syncretism. (Child and Child 1985) challenge the concept of female pollution in Balzer's theory, since it would present a negative view of menstruation that is not supported by "the material she presents" and would reveal instead a projection of Balzer's own cultural values. Balzer's response is that this negative view is present among Khanty women, as documented by practices (e.g., female exclusion to menstrual huts) and semantic analyses of Khanty vocabulary on menstruation. Other ethnographies would confirm her interpretation. (Balzer 1981) also warns that anthropologist should not fall into "reverse ethnocentrism traps" by wishing that menstruation were viewed more positively in other cultures than in the anthropologist's own. In other words, (Child and Child 1985) would challenge the use of a negatively connoted Western theoretical concept (pollution) on Khanty material. (Balzer 1981) defends this use as appropriate given the negative connotation menstruation has among the Khanty. In other words, for Balzer one 
abides by cultural relativism to the extent that there is a match between the negative values in the source and the target culture. Seeking any mismatch (via positive connotations) would reveal instead, for Balzer, an ethnocentric slip. Who is interpreting the standard correctly here?

(Foin and Davis 1987) use data from Maring ethnographies to test formal models of human population dynamics. (LiPuma 1988) accuses them of ethnocentrism for understanding Maring social practices in terms of their own "Western materialistic ideology", since (Foin and Davis 1987) would explain emigration by causal factors (number of pigs) that are not reflected on Maring "cultural concepts and practices" on emigration. (Davis and Foin 1988) reply makes explicit that they are just formalizing other anthropologists' work without assessing their assumptions. Yet, they suspect, LiPuma's objection are not about the details of their model, but rather about mathematical modelling in anthropology. In my own words, imposing (Western) causal structures on (non-Western) holistic webs of practices would count, for LiPuma, as a breach of cultural relativism. For Davis and Foin, this is just a methodological disagreement on explanation. Again, it is far from clear who is interpreting the standard correctly here.

These two examples illustrate very abstract forms of (purported) ethnocentrism, where the cultural prejudice lies in a particular theoretical standpoint. All the involved parties adhered to the standard of cultural relativism, but this shared commitment does very little to close the conflict, precisely because there was no agreement about what counted as bias. Both parties can see each other as biased by different parts of their own culture, triggering debates that are impossible to close on the basis of the cultural relativism standard alone since there is 
no consensual authority to adjudicate it. If cultural relativism was born in the context of a debate on the purported superiority of some cultural forms, we may conclude that, on its own, it did very little to close it.

\section{Concluding Remarks: Why Not Rules Instead?}

I have argued that cognitive biases are controlled with methodological norms of, at least, two formats: rules and standards. In experimental disciplines with a causal approach, debiasing rules seem easier to formulate and implement, because they target the particular points in the data gathering process in which bias may emerge, prescribing definite actions to control for them. In non-causal forms of research, like participant observation in anthropology, cognitive biases appear as interpretative slips, for which there are no fixed ex ante corrections. Methodological norms like cultural relativism are formulated as standards instead, creating the problem of adjudicating them for particular pieces of evidence. As we just saw in the previous section, anthropologists do not seem to agree on how to carry out this adjudication process, whereas rule-based experimental fields exhibit, prima facie, a substantive consensus on biases and debiasing methods. In pharmacology, a field fraught with financial conflicts of interest professional bodies like the Cochrane collaboration have produced reference lists of biases and debiasing methods to score the quality of trial evidence that are regarded as a benchmark and elicit a broad professional consensus as to what counts as good trial evidence (Sterne et al. 2019). Instead, the American Anthropological Association, perhaps still faithful to Boas, has just generically promoted cultural relativism, without any guideline about how to detect it and correct it. 
We may finally wonder why it is that this lack of consensus on ethnocentrism does not apparently hinder research in anthropology. In my view, there are two complementary reasons. On the one hand, cultural relativism may be a simple guideline for reporting, more than an actual correction for any bias. On the other hand, in as long as anthropologists do not build up generalizations, they do not need to rely on each other's reports, aggregating their evidence.

Cultural relativism maybe just a guideline in the process of generating ethnographic evidence: it is about not expressing dismay whenever confronted by practices that struck the anthropologist as illogical or repugnant (Brown, xa, and F 2008, 367). However, the anthropologist may entertain such thoughts in private, provided they are not included in the field notes ore the published report. A notorious case in point was Bronislaw Malinowski whose private records about his experience with the Trobrianders are significantly more ethnocentric than his famous monograph (Malinowski 1989). Nonetheless, the latter were considered for decades masterpieces of fieldwork. However, even as reporting guideline, cultural relativism may be challenged. Colin Turnbull famously lost his "anthropological cool" during his time with the Ik, a severely deprived culture in Uganda in which there was very little cooperation among its members in order to avoid any sharing of their scarce resources. Turnbull not only reported his shock in print, but recommended that the Ik were disbanded and its members incorporated into more socially cohesive cultures (Turnbull 1972). For many anthropologists, Turnbull was guilty of breaching cultural relativism, contaminating his report with his own value judgments (Wilson et al. 1975). But Turnbull defended his report arguing that the anthropologist "should present his involvement as part of the data", so 
the reader can assess the involvement itself "as data" and discount it like any other characteristic of the ethnographer (Wilson et al. 1975, 356). Perhaps the lack of professional consensus as to what counts as ethnocentrism is not a problem because each individual anthropologist can read this way other colleague's work. I.e. discounting what in her own definition counts as biased in a report and building up on whatever else is left.

This should not be a problem to the extent that anthropologists can dispense with consensual forms of evidence aggregation. Again, the comparison with pharmacology is useful: having a standardized score of the risk of bias in a clinical trial (depending on whether it is randomized, blinded, etc.) is crucial for aggregating evidence from different trials into meta-analyses. Here, the risk of bias in a dataset can be transformed into a numerical weight that will ponder the influence of each single trial in the aggregated conclusions about treatment effects. Anthropology has instead evolved into more and more extreme forms of particularism (Jarvie 1993): each ethnography reports about the events a particular anthropologist witnessed without any pretence of generality as to what happened before (or will happen after) the fieldwork. For some, an ethnocentric ethnography may be a case of professional misconduct, but no data point is not lost for further analysis. If at any point, there is a paradigm shift in anthropology such that generalization and comparison become acceptable again, it will be probably be useful to transform the current standard against ethnocentrism into a set of debiasing rules capable of adjudicating debates. After all, several rules of thumb seem to be already at work among practitioners (length of the field visit, command of the language, systematicity of the field notes, etc.). Transforming these informal 
prescriptions into formalized debiasing rules, conventional as they may be, will be more effective at controlling for cognitive biases than the current standard on cultural relativism. 
References

Balzer, Marjorie Mandelstam. 1981. "Rituals of Gender Identity: Markers of Siberian Khanty Ethnicity, Status, and Belief." American Anthropologist 83 (4): 850-867

Boas, Franz. [1932] 1940. "The aims of anthropological research." In Race, language and culture, edited by Franz Boas, 243-259. New York,: The Macmillan company.

---. [1936] 1940. "History and science in anthropology: a reply." In Race, language and culture, edited by Franz Boas, 305-315. New York,: The Macmillan company.

Boas, Franz, and Ruth Benedict. 1938. General anthropology. Boston, New York etc.: D.C. Heath and Company.

Brown, Michael, xa, and F. 2008. "Cultural Relativism 2.0." Current Anthropology 49 (3): 363383.

Chalmers, Iain. 2006. "Why fair tests are needed: a brief history." Evidence Based Medicine 11 (3): 67-8.

Child, Alice B., and Irvin L. Child. 1985. "Biology, Ethnocentrism, and Sex Differences." American Anthropologist 87 (1): 125-128.

Davis, William G., and Theodore C. Foin. 1988. "Equilibrium Unconsidered." American Anthropologist 90 (4): 973-976.

Foin, Theodore C., and William G. Davis. 1987. "Equilibrium and Nonequilibrium Models in Ecological Anthropology: An Evaluation of "Stability" in Maring Ecosystems in New Guinea." American Anthropologist 89 (1): 9-31.

Fon, Vincy, and Francesco Parisi. 2007. "On the optimal specificity of legal rules." Journal of Institutional Economics 3 (2): 147-164.

Fugelsang, J., C. Stein, A. Green, and K. Dunbar. 2004. "Theory and data interactions of the scientific mind: Evidence from the molecular and the cognitive laboratory." Canadian Journal of Experimental Psychology 58: 132-141.

Hatch, Elvin. 1983. Culture and morality : the relativity of values in anthropology. New York: Columbia University Press.

Herskotvits, Melville J. [1955] 1973. "Cultural relativism and cultural values." In Cultural relativism: perspectives in cultural pluralism, 11-34. New York: Vintage Books.

---. [1958] 1973. "A cross-cultural view of bias and values." In Cultural relativism: perspectives in cultural pluralism, 97-109. New York: Vintage Books.

---. [1960] 1973. "The humanism in anthropological science " In Cultural relativism: perspectives in cultural pluralism, 242-263. New York: Vintage Books.

Jarvie, I.C. 1993. "Review Essays : Relativism Yet Again." Philosophy of the Social Sciences 23 (4): 537-547.

Koskinen, Inkeri. 2019. "Relativism in the Philosophy of Anthropology." In The Routledge Handbook of Philosophy of Relativism, edited by Martin Kusch, 45-55. Routledge.

LiPuma, Edward. 1988. "Ethnographic Equilibrium." American Anthropologist 90 (4): 970973. 
Luppi, Barbara, and Francesco Parisi. 2011. "Rules versus Standards." In Encyclopedia of Law and Economics, edited by Gerrit De Geest. Cheltenham, UK: Edward Elgar

Malinowski, Bronislaw. 1989. A diary in the strict sense of the term. Stanford, Calif.: Stanford University Press.

Staley, Kent W. 2004. The evidence for the top quark: objectivity and bias in collaborative experimentation. Cambridge: Cambridge University Press.

Sterne, Jonathan AC, Jelena Savović, Matthew J Page, Roy G Elbers, Natalie S Blencowe, Isabelle Boutron, Christopher J Cates, Hung-Yuan Cheng, Mark S Corbett, and Sandra M Eldridge. 2019. "RoB 2: a revised tool for assessing risk of bias in randomised trials." BMJ 366:14898.

Teira, D. 2016. "Debiasing methods and the acceptability of experimental outcomes." Perspectives on Science 24 (6): 722-743.

Turnbull, Colin M. 1972. The mountain people. New York: Simon and Schuster.

Weber, Elke U., and Eric J. Johnson. 2009. "Mindful Judgment and Decision Making." Annu. Rev. Psychol. (60): 53-85.

Wilson, Peter J., Grant McCall, W. R. Geddes, A. K. Mark, John E. Pfeiffer, James B. Boskey, and Colin M. Turnbull. 1975. "More Thoughts on the Ik and Anthropology [and Reply]." Current Anthropology 16 (3): 343-358.

Zahle, Julie. 2013. "Participant Observation and Objectivity in Anthropology." In New Challenges to Philosophy of Science, edited by H. Andersen, D. Dieks, W. J. Gonzalez, T. Uebel and G. Wheeler, 365-376. Springer. 